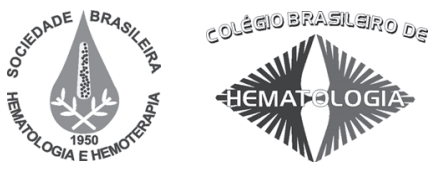

Revisão / Review

\title{
O G-CSF na terapia do acidente vascular cerebral
}

\section{The potential role of $G$-CSF in stroke}

\author{
Angelo L. Maset ${ }^{1}$ \\ Lilian Piron-Ruiz ${ }^{2}$ \\ Oswaldo T. Greco ${ }^{3}$ \\ Mario Lago ${ }^{4}$ \\ Alana F. C. Poloni ${ }^{5}$ \\ Milton A. Ruiz ${ }^{6}$
}

\begin{abstract}
O fator estimulador de colonias granulocitárias (G-CSF) é uma glicoproteína descrita há mais de vinte anos, e é largamente utilizada para tratamento de estados neutropênicos e no transplante de medula óssea. O G-CSF estimula células-tronco hematopoéticas e regula crucialmente a sobrevivência de neutrófilos maduros, pósmitóticos, através da inibição da apoptose. Além do efeito sistêmico, mais recentemente tem-se demonstrado uma surpreendente atividade do G-CSF no sistema nervoso central. A administração de G-CSF mobiliza células-tronco e progenitoras da medula óssea para o sangue periférico, que, por sua vez, atravessa a barreira hemato-encefálica (BHE) e se dirige à área acometida do cérebro. A atividade do GCSF no sistema nervoso central tem sido caracterizada como multimodal, pois, além do efeito mobilizador de células da medula óssea, demonstrou uma ação direta neuroprotetora através de diferentes mecanismos, tais como a atividade antiapoptótica em neurônios, regeneração da vascularização, efeito anti-inflamatório e estimulação da neurogênese endógena. Este relato sumariza a ação do G-CSF no sistema nervoso central e aborda seu potencial para o emprego no acidente vascular cerebral. Rev. Bras. Hematol. Hemoter. 2009;31(Supl. 1):104-111.
\end{abstract}

Palavras-chave: G-CSF; isquemia; isquemia cerebral; AVC isquêmico; neuroproteção.

\section{Introdução}

Existem várias abordagens utilizando terapia celular para vários tipos de acometimentos do SNC em curso neste momento. ${ }^{1-8}$ Uma delas é a utilização do fator estimulador de células granulocitárias (G-CSF) como fator mobilizador de células-tronco endógenas. $\mathrm{O}$ princípio da terapia celular no SNC é o de restaurar a função do cérebro humano danificado pelos diversas doenças, restaurando os tipos celulares neurais afetados por novas células ou protegendo as células neurais ainda presentes, interrompendo o processo de perda neuronal e minimizando assim a perda funcional cerebral. $\mathrm{Na}$ complexa cascata de eventos fisiopatológicos associada com a isquemia cerebral aguda, uma abordagem multimodal, isto é, uma abordagem que atuasse simultaneamente em múltiplas vertentes desta cascata teoricamente teria mais chance de sucesso do que abordagens isoladas.

\section{Neurogênese na isquemia cerebral}

A hipótese de uma ação benéfica do G-CSF no acidente vascular cerebral (AVC) está intimamente ligada à neurogênese na isquemia cerebral e, para compreender o seu mecanismo de ação, precisamos compreender a resposta fisio-

\footnotetext{
${ }^{I}$ Neurocirurgião. Médico Research Fellow MCV - USA.

${ }^{2}$ Médica Assistente do Serviço de Transplante da Faculdade de Medicina da USP-SP.

${ }^{3}$ Cardiologista. Instituto de Moléstias Cardiovasculares (IMC) - São José do Rio Preto-SP.

${ }^{4}$ Biólogo do IMC, São José do Rio Preto-SP.

${ }^{5}$ Engenheira - Coordenadora de Banco de Dados de Terapia Celular do IMC, São José do Rio Preto-SP.

${ }^{6}$ Professor colaborador da Disciplina de Hematologia e Hemoterapia da FMUSP-SP e coordenador do Grupo de Terapia Celular

do IMC/HMC - São José do Rio Preto-SP.
}

Grupo de Estudos em Terapia Celular do Instituto de Moléstias Cardiovasculares (IMC), São José do Rio Preto-SP.

Correspondência: Angelo Luiz Maset

Av Francisco C Oliveira 1100 - Jardim Morumbi

15090-190 - São José do Rio Preto-SP

E-mail:maset@terra.com.br

Doi: 10.1590/S1516-84842009005000022 
lógica ao AVC. No início desta década, vários estudos demonstraram que os precursores neurais endógenos iniciam uma resposta compensatória ao AVC que resulta na produção de novos tipos celulares, ocorrendo este fenômeno inclusive no cérebro adulto.

Jin e colaboradores, ${ }^{9}$ em 2001, demonstraram em modelos focais de isquemia cerebral onde existe dano no hemisfério ipsilateral à oclusão arterial, que a incorporação de bromodeoxiuridina (BrdU) aumentou em oito vezes na zona subgranular (SGZ) ipsilateral sete dias após a isquemia quando comparado com o grupo controle. Surpreendentemente, a SGZ contralateral também aumentou em quatro vezes a incorporação de BrdU quando comparado ao grupo controle, persistindo a marcação duas semanas após o ictus isquêmico.

Nakatomi e colaboradore ${ }^{10}$ demonstraram a existência de regeneração de células piramidais no hipocampo. Importante, no entanto, foi a demonstração de que estas células regeneradas se integraram ao circuito neuronal, sendo isto um pré-requisito para a restauração da função cerebral. Eles estudaram as propriedades eletrofisiológicas das novas sinapses, através das colaterais de Schaffer, 120 dias após isquemia cerebral em três grupos distintos de ratos: um grupo controle, um grupo somente com isquemia e um terceiro grupo submetido à isquemia cerebral e tratado com fator de crescimento de fibroblastos (FGF) imediatamente após a isquemia cerebral ter sido provocada. Os resultados demonstraram que ocorreram potenciais pós-sinápticos excitatórios com marcada atenuação nos animais que sofreram isquemia (ambos os grupos). Observou-se, no entanto, uma atenuação bem menor nos animais tratados com FGF; o slope da curva dos animais tratados com FGF se aproximou do grupo controle a tal ponto que não houve diferença estatística. Este trabalho demonstrou a grande capacidade regenerativa de células progenitoras neurais piramidais no cérebro adulto.

Arvidsson, do grupo de Lindvall, ${ }^{11}$ demonstrou que o acidente vascular cerebral isquêmico (AVCi), causado pela oclusão transitória em ratos adultos, causou um aumento considerável na proliferação celular na zona subventricular. Os novos neurônios gerados após o AVCi migraram para a área lesada do estriado, onde expressaram marcadores para neurônios maduros e em desenvolvimento. Assim, houve a comprovação de que o AVCi induziu a diferenciação de novos neurônios com o fenótipo da maioria dos neurônios destruídos.

Este dado comprovou que o cérebro adulto possui capacidade de autorreparo após a lesão causadora de morte neuronal. Assim, se ocorre proliferação celular em determinados sítios anatômicos após o AVCi, e se estes neurônios migram para a região do ictus isquêmico, diferenciando-se com a expressão fenotípica dos neurônios lesados, cabe a pergunta: por que estes pacientes não se recuperam? Aparentemente, um dos motivos é o baixo porcentual de neurônios sobreviventes. ${ }^{11}$ Os dados sugerem que $80 \%$ dos no- vos neurônios gerados pela resposta compensatória neural morrem durante as seis primeiras semanas após o ictus isquêmico, e somente $0,2 \%$ das células lesadas são respondidas pela neurogênese. A neurogênese induzida pelo AVC era considerada como uma resposta aguda e transitória até 2006, quando Thored, ${ }^{12}$ também do grupo de Lindvall, demonstrou que os neuroblastos estriatais são gerados sem declínio por quatro meses após o AVC em ratos adultos. Os novos neuroblastos assim formados precocemente ou tardiamente formaram neurônios maduros que sobreviveram por vários meses. Esta resposta celular foi coincidente com a melhora funcional. Houve uma melhora funcional inicial nos testes da escada e do cilindro, que se estabilizou após um mês, mas houve uma melhora acentuada na marcha entre o primeiro e o quarto mês após o AVC utilizando-se o teste da grade. Assim, o grupo de Lindvall ${ }^{11,12}$ forneceu as primeiras evidências de que o cérebro adulto pode responder ao infarto cerebral gerando novos neurônios por períodos prolongados.

\section{Terapia celular e o G-CSF}

A terapia celular do G-CSF se utiliza da abordagem endógena. Conceitualmente, a abordagem endógena faz uso da população de NSC's naturalmente presente no sistema neural do paciente, mobilizando células precursoras e progenitoras e potencializando uma resposta compensatória existente. Com esta assertiva torna-se compreensível e atraente a estratégia de administrar agentes mobilizadores de células precursoras que amplificarão as células precursoras endógenas, fornecendo uma via alternativa de reinervação do cérebro danificado e potencializando a resposta compensatória. A mobilização de células precursoras é menos problemática em todos os aspectos quando comparada com o transplante, e possui uma sólida base científica. ${ }^{10,13-22}$

\section{O G-CSF como agente mobilizador e protetor}

Dentre os vários agentes mobilizadores de células precursoras conhecidos atualmente, o G-CSF tem recebido considerável atenção. O G-CSF é uma glicoproteína com 19,6 kilodaltons e membro da família de citocinas de fatores de crescimento, descrita há mais de vinte anos, inicialmente como uma indutora da diferenciação da célula leucêmica monocitária WEHI-3B, ${ }^{23,24} \mathrm{e}$ clonada por Nakata. ${ }^{25}$ Nicola e colaboradores ${ }^{26}$ caracterizaram os receptores do G-CSF em humanos e, posteriormente, outros autores detalharam as suas características bioquímicas e moleculares. ${ }^{27,28} \mathrm{O}$ receptor do G-CSF é típico das citocinas (CD114) com um domínio de transmembrana e de transdução de sinal intracelular. Incluem-se como fontes do G-CSF os monócitos, as células mesoteliais, os fibroblastos e as células endoteliais. O G-CSF estimula o crescimento de precursores de neutrófilos e regula crucialmente a maturação e a sobrevivência da linhagem de granulócitos neutrófilos maduros, pós-mitóticos, através 


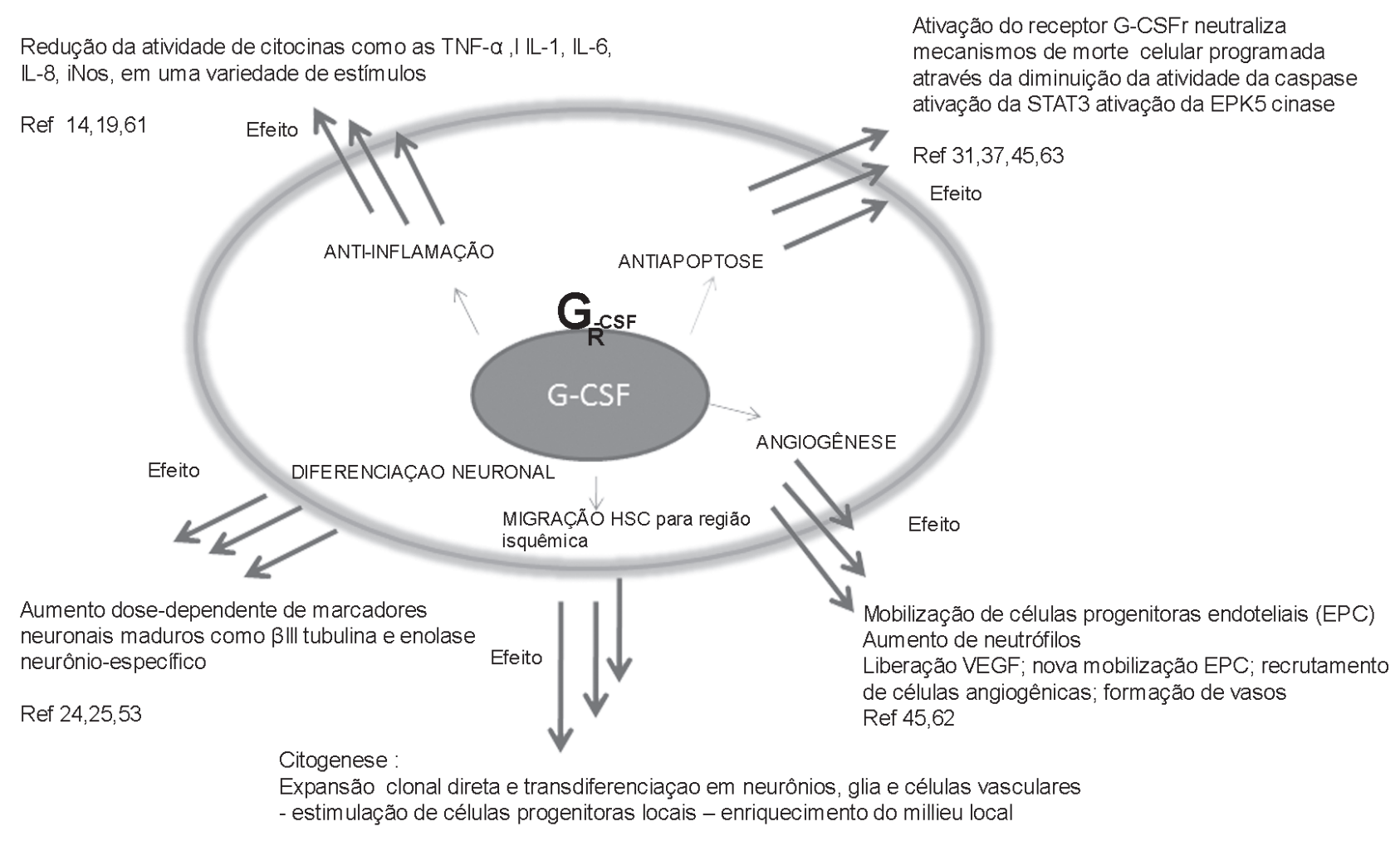

Ref $57,59,67$

Figura 1. Efeito multimodal do G-CSf no SNC

da inibição da apoptose. ${ }^{15,23,29}$ Atualmente ele é utilizado para tratar neutropenia induzida por agentes quimioterápicos, ou no transplante de medula óssea. ${ }^{23-25,30-33} \mathrm{O}$ G-CSF é uma droga liberada pelo FDA e pelo Ministério da Saúde no Brasil, tendo já sido administrada a milhões de pacientes em todo o mundo, e é bem tolerada pelo organismo humano. Mais recentemente, tem sido demonstrada uma surpreendente atividade do G-CSF no SNC, ${ }^{13,17,20,22,34-38}$ fato que adiciona um potencial regenerativo em várias doenças neurológicas. A administração do G-CSF mobiliza células-tronco e progenitoras da medula óssea para o sangue periférico, as quais, por sua vez, ultrapassam a barreira hematoencefálica e se dirigem à área acometida do cérebro. Além deste papel periférico, o G-CSF e seus receptores se expressam em grande parte do tecido neural, o que justificaria o seu efeito neuroprotetor aparentemente multimodal (Figura 1), incluindo-se uma atividade antiapoptótica nos neurônios, ${ }^{16,20,22}$ regeneração e reparação da vascularização e estímulo da angiogênese, ${ }^{17}$ além de um efeito anti-inflamatório ${ }^{39}$ e do estímulo da neurogênese endógena. $^{21}$

$A$ atividade protetora do G-CSF após a isquemia cerebral

Konishi ${ }^{40}$ descreveu os efeitos tróficos do G-CSF em células neuronais, mas $\mathrm{Schabitz}^{20} \mathrm{e} \mathrm{Six}^{38}$ foram os primei- ros a descrever a atividade protetora na isquemia cerebral. Num modelo de infarto cerebral de artéria cerebral média (ACM) em ratos, eles demonstraram que o G-CSF reduziu o tamanho do infarto cerebral induzido pelo glutamato em $47 \% .{ }^{20}$ Além disso, o G-CSF também reduziu significativamente a mortalidade (25\% no grupo controle versus $6,25 \%$ no grupo do G-CSF). Num estudo subsequente, ${ }^{22}$ o grupo de Schabitz demonstrou o potencial terapêutico multimodal do G-CSF; eles demonstraram a forte atividade antiapoptótica em neurônios maduros, através da ativação de múltiplas vias de sobrevivêncica celular. Ambos - G-CSF e o seu receptor (G-CSFr) - se expressaram em inúmeros sítios anatômicos e funcionais do $\mathrm{SNC}$, e esta expressão foi induzida pela isquemia cerebral, o que sugeriu um mecanismo sinalizador de proteção autócrino. Surpreendentemente, o G-CSFr também se exprimiu nas células-tronco neurais adultas, e o G-CSF induziu a diferenciação neuronal in vitro. O G-CSF melhorou significativamente o resultado comportamental de longo prazo e, portanto, melhorou o prognóstico de longo prazo após a isquemia cortical, estimulando a resposta progenitora neural e propiciando assim uma nova oportunidade de recuperação funcional. Os autores concluiram que o G-CSF é um ligante endógeno no SNC que possui uma atividade benéfica dupla, seja agindo contra a degeneração neuronal aguda ou contri- 
buindo para uma plasticidade de longo prazo após a isquemia cerebral.

Schneider e Schabitz ${ }^{7}$ recentemente se utilizaram de dois modelos de isquemia cerebral em ratos; no primeiro eles mensuraram o volume do infarto cerebral num modelo de isquemia de oclusão da ACM, e estenderam o início do tratamento com G-CSF após a isquemia para 24 horas e 72 horas após o ictus isquêmico num modelo de isquemia fototrombótica cortical, analisando separadamente os efeitos da isquemia e do G-CSF no córtex cerebral e em regiões subcorticais, e demonstraram que a eficácia do G-CSF se estende para as áreas cortical e subcortical. Até aquele momento, todas as drogas conhecidas tinham eficácia apenas no córtex cerebral. Assim como eles, outros autores demonstraram que a janela terapêutica após o infarto pode ser bastante flexível, pois o G-CSF teria uma eficácia verdadeiramente bimodal (antiapoptóptica na fase aguda e potencializadora da recuperação na fase subaguda e crônica). Outros autores, como Six e colaboradores,$^{38}$ demonstraram uma redução de $55 \%$ na área do infarto com janela terapêutica de 24 horas $(n=6)$. Komine-Kobayashi e colaboradores $^{36}$ demonstraram uma redução equivalente em dois grupos de animais, um de 24 horas e outro de 72 horas após o infarto. Esta ação bimodal torna o G-CSF um candidato extremamente atraente para aplicação clínica na fase subaguda do AVC. Schabitz, ${ }^{20} \mathrm{Zhao}^{41}$ e $\mathrm{Lu}^{19}$ também demonstraram que modelos experimentais tratados com G-CSF tiveram melhor recuperação funcional, menor taxa de mortalidade e menor volume de infarto.

Kawada e colaboradores ${ }^{35}$ exploraram o potencial do G-CSF na fase aguda (1-10 dias) e subaguda (11-20 dias) do AVC e mostraram que o G-CSF atuou de uma maneira distinta, pois, na fase subaguda, o G-CSF também melhorou as funções cerebrais cognitivas.

O G-CSF mobiliza as células progenitoras hematopoéticas (HSCs) para o SNC

HSCs mobilizadas da medula óssea (CD34+) pelo GCSF migram através da circulação e atravessam a BHC; elas podem ser observadas nos espaços perivasculares do hemisfério isquêmico. ${ }^{42}$ Embora haja uma melhora no processo de reparo celular, o mecanismo exato de como este fato acontece ainda não é compreendido. Existem alguns processos que são conhecidos. Sabemos que a isquemia causa um aumento na expressão de receptor de citocinas CXCR4 SDF-1 em regiões adjacentes à isquemia, indicando que o SDF-1 pode exercer quimioatração às células $\mathrm{CD} 34+\mathrm{CXCR} 4$ periféricas. Assim, o SDF-1 seria um sinalizador molecular que direcionaria as células CD34+ CXCR4 para a área isquêmica. ${ }^{12,43}$ Os mecanismos que gerariam o reparo celular (refletidos como um aumento em sobrevida e melhoria nas escalas de avaliação clínica) são mais especulativos. Uma possibilidade seria a integração ao tecido das células CD34 mobilizadas e que atingiram a área isquêmica e de penumbra, repondo as células lesionadas e reconstruindo o circuito neuronal. Uma outra possibilidade seria a produção de fatores tróficos a partir da interação das células CD34 com as células isquêmicas, e estes fatores tróficos contribuiriam para a reparação das células lesadas e ainda viáveis. Zhao ${ }^{41}$ mostrou que os níveis de fibronectina no cérebro de ratos tratados com GCSF estão elevados, e que a fibronectina promove a sobrevivência e migração de células precursoras neurais endógenas transplantadas em cérebros de ratos com traumatismo craniencefálico. Modelos experimentais com camundongos deficientes em fibronectina possuem uma atividade apoptótica e área de infarto muito superior após a oclusão da ACM quando comparados com camundongos normais.

\section{O G-CSF ativa os mecanismos de antiapoptose}

O G-CSF se mostrou um forte ativador de vias de sobrevivência celular (antiapoptose); esta propriedade parece ser mediada pelo receptor neuronal G-CSF (G-CSFr). Uma via antiapoptótica conhecida é a regulação da STAT-3 e Bcl2, ${ }^{36}$ família de protooncogenes que promove a sobrevivência celular. Outra possibilidade seria através da via da proteína Janus tirosina quinase /transdutor e ativador de sinal de transcrição (JAK/STAT) e subsequente ativação da Bcl-2, ${ }^{42,44}$ onde a hiperexpressão de $\mathrm{Bcl}-2$ protegeu os neurônios contra a morte no período pós-isquemia. O grupo de Schabitz ${ }^{22}$ demonstrou a existência de G-CSFr em neurônios e na glia, e que o efeito neuroprotetor do G-CSF é dependente da ativação da via JAK/STAT. Schabitz, ${ }^{20} \mathrm{Zhao}^{41} \mathrm{e} \mathrm{Lu}^{19}$ demonstraram que modelos experimentais tratados com G-CSF tiveram melhor recuperação funcional, menor taxa de mortalidade e menor volume de infarto.

\section{O G-CSF induz a diferenciação neuronal}

Sabe-se que o cérebro dos mamíferos adultos possuem células-tronco e progenitoras em vários sítios, incluindose a zona subventricular e giro denteado. O G-CSF e seu receptor se expressam também nestas regiões. ${ }^{22} \mathrm{O}$ G-CSF induz a atividade do marcador neuronal maduro B-III tubulina, indução esta que é mais intensa do que a maioria dos indutores conhecidos. O G-CSF leva a um aumento da população de células neurais que expressam marcadores neuronais maduros, sendo este dado uma indicação de que o G-CSF modula a diferenciação de células precursoras neurais adultas. ${ }^{22}$ O G-CSF também estimula a neurogênese através da integração com o fator de crescimento vascular endotelial (VEGF) e ativação de estatinas. ${ }^{16}$ A administração de citocinas hematopoéticas na fase subaguda após o infarto cerebral foi eficaz na recuperação funcional, facilitando a proliferação de células progenitoras neurais. ${ }^{35}$

\section{O G-CSF interfere na angiogênese}

Concomitante ao aumento dos granulócitos e dos neutrófilos na circulação sanguínea, o G-CSF promove a secreção de fatores angiogênicos, ${ }^{45}$ como o VEGF, utilizando- 
se das alternativas de aumentar o número absoluto de neutrófilos e do aumento da produção de VEGF em cada neutrófilo. O bloqueio das vias de VEGF impede a angiogênese induzida pelo G-CSF, sugerindo que a angiogênese induzida pelo G-CSF é dependente de VEGF. ${ }^{45} \mathrm{~A}$ administração local de G-CSF no tecido isquêmico elevou a densidade capilar e forneceu uma vascularização funcional, contribuindo para a neovascularização do tecido isquêmico. ${ }^{17} \mathrm{Nes}-$ ta situação observou-se área vascular com aumento de ramificações, do comprimento dos vasos e do número de células endoteliais BrdU positivas no grupo tratado com GCSF quando comparadas com o grupo isquêmico. Mas esta não é a única forma de ação, pois existem evidências de que células circulantes angiogênicas são capazes de se dirigir para locais de dano vascular estimulando a angiogênese, muito embora o número destas células angiogênicas circulantes seja considerado muito pequeno. Takagi ${ }^{46}$ demonstrou um aumento do índice de perfusão sanguínea, além do aumento do número de vasos colaterais mensuráveis e da densidade capilar por meio de Doppler. A combinação de transplante de células mononucleares de medula óssea e do G-CSF apresentou o melhor porcentual de neovascularização. Um fator que pode interferir na eficácia do G-CSF como indutor de angiogênese é o fator tempo. O G-CSF injetado um dia após a isquemia cerebral induziu a uma maior taxa de proliferação endotelial quando comparado com injeções ocorridas sete dias após a isquemia. ${ }^{17}$ Aparentemente, o G-CSF também atua na diferenciação de células HSC's, diferenciando-as em células endoteliais de vasos sanguíneos. Minamino ${ }^{47}$ observou que esta diferenciação estava aumentada em animais tratados com G-CSF, resultando numa recuperação mais precoce do fluxo sanguíneo em membros isquêmicos ${ }^{47}$

\section{O G-CSF inibe os mediadores inflamatórios}

Os antígenos que penetram o corpo humano são combatidos pelo sistema imunológico inespecífico do qual fazem parte os granulócitos (PMN) e os macrófagos, entre outros. No bojo desta batalha contra a infecção aparecem os elementos que definem a inflamação, como a hiperemia, o edema e a hipertermia local. Nosso sistema de defesa deste componente do sistema imunológico é coordenado e regulado através de padrões de sinalização celular (molecular), por mediadores lipídicos e pelas citocinas interleucina-1(IL-1), fator de necrose tumoral alfa (TNF- $\alpha$ ) e a linfocina interferon gama (IFN- $\gamma$ ). A atividade destas citocinas pode ser reduzida não somente pela down-regulation de sua produção ou secreção, mas também pela estimulação da liberação de seus antogonistas IL-ra e sTNF- $\alpha$. Pois é neste contexto que o G-CSF age: o G-CSF ativa os PMN, estimulando a sua proliferação, maturação e direcionamento para defesa. Assim o G-CSF pode ser um potente imunomodulador anti-inflamatório por si só. Então, à luz destes dados, o próximo passo seria conhecer com mais detalhes os efeitos do G-CSF nestes mediadores, pois drogas que interferem na produção ou na ação destes mediadores potencialmente fornecem proteção contra a inflamação. Um importante denominador comum de muitas condições inflamatórias é a liberação de TNF. Drogas que interferem na produção ou na ação do TNF potencialmente fornecem proteção contra a inflamação. $O$ G-CSF reduz a atividade do $\mathrm{TNF} \alpha,{ }^{48}$ inibe a atividade da enzima óxido nítrico sintase induzível (iNOs), ${ }^{49}$ bem como diminui também os níveis de IL-1 $\beta$, IL-6, IL-8 em várias condições. ${ }^{50}$ Ekdahl, ${ }^{51}$ do grupo de Lindvall, demonstrou que a inflamação artificialmente induzida por lipopolissacarídeos (LPS) de Escherichia coli piora tanto a neurogênese basal quanto a induzida pela injúria. Os autores propuseram que a inflamação aumenta a ativação microglial, e que este seria o fator primordial da inibição da neurogênese. Este efeito deletério da microglia ativada dos neurônios justaformados é mais provavelmente mediado através da ação de citocinas, tais como a IL-1 $\beta$ ou IL- 6 , TNF- $\alpha$, ou óxido nítrico (NO). Estas moléculas podem ser liberadas na microglia e são neurotóxicas in vitro ${ }^{33,52,53,54}$ Monje e colaboradore ${ }^{55}$ demonstraram que a inflamação produzida isoladamente no SNC inibe a neurogênese, e que o bloqueio do processo inflamatório com indometacina restaura a neurogênese após a inflamação induzida por endotoxina ou após irradiação cerebral. Os autores também discutem a importância e os possíveis mecanismos na fisiopatologia da memória e em certas demências.

\section{Implicações clínicas dos estudos em humanos}

Os estudos promissores realizados em animais deram o embasamento científico para o uso clínico do G-CSF em humanos. As estratégias de reposição celular em humanos se iniciaram com o transplante intracerebral de células fetais ou células precursoras pré-diferenciadas in vitro. ${ }^{3,6,56}$ Estas estratégias concebiam uma abordagem em uma única via na cadeia de eventos que levam à morte celular. O G-CSF propicia uma nova terapia, pois as várias publicações existentes, como já citamos anteriormente, demonstram um efeito multimodal do G-CSF, atuando tanto na fase aguda quanto na fase subaguda da isquemia cerebral. O G-CSF melhorou o resultado comportamental de longo prazo após a isquemia cortical. Assim, aparentemente, o G-CSF exerce uma atividade benéfica dupla, ou seja, agindo contra a degeneração neuronal aguda ou contribuindo para uma plasticidade ${ }^{13,19,49}$ de longo prazo após a isquemia cerebral. O G-CSF preenche os critérios do STAIR, ${ }^{62}$ quais sejam: penetração na barreira hemato-encefálica, atividade neuroprotetora em diferentes modelos de infarto cerebral demonstrada por grupos independentes, atividade demonstrada em diferentes espécies, farmacocinética bem conhecida, e dados prognósticos promissores.

Ickenstein, ${ }^{57} \mathrm{em} 2004$, reportou a ausência de efeitos adversos do G-CSF; em 2006, Shyu ${ }^{37}$ e colaboradores exploraram o potencial terapêutico do G-CSF no AVC isquêmico 
agudo da artéria cerebral média avaliando dez pacientes que tiveram AVC recente. Os critérios de inclusão foram: diagnóstico através de ressonância magnética à admissão hospitalar, déficit neurológico avaliado pela escala de stroke do NIH (NIHSS), pacientes selecionados quando entre $9 \mathrm{e}$ 20 desta escala, e período máximo de sete dias após início do AVC. Num período de 12 meses após o tratamento, sete pacientes que receberam G-CSF apresentaram melhora muito mais expressiva na função neurológica do que os três pacientes-controle em todas as escalas clínicas utilizadas, incluindo a Escala de Stroke do NIH, Escala de Stroke Européia, e o índice de Barthel. Estes resultados positivos encorajadores estimularam a realização de novos estudos clínicos com um maior número de pacientes e dentro de padrões estatísticos inquestionáveis. Sprigg e colaboradores ${ }^{58} \mathrm{pu}-$ blicaram, em 2006, um estudo duplo-cego, aleatório e controlado com placebo em 36 pacientes com AVC isquêmico, realizado em dois centros, onde foi aplicado G-CSF subcutâneo com aumento escalonado de dose, na fase subaguda (de sete a trinta dias). Foi realizada a contagem de células $\mathrm{CD} 34^{+}$e hematimétrica global para se atestar a mobilização de células precursoras, sendo o objetivo do experimento clínico avaliar a segurança e o resultado funcional dos pacientes. Os autores concluiram que o G-CSF é eficaz na mobilização de células precursoras em pacientes com AVC isquêmico recente, e que a administração é segura e bem tolerada. Apesar do início promissor, novos estudos deverão agora avaliar a eficácia da droga como agente terapêutico no AVC. Existem ainda vários quesitos a serem respondidos, como, por exemplo, o que aborda a questão da dosagem mais eficaz. Quais são os limites temporais da eficácia do G-CSF após o AVC? É possível potencializar ainda mais a ação do G-CSF aplicando-o simultaneamente com outras citocinas, tais como fator neurotrófico derivado do cérebro (BDNF), fator derivado vascular endotelial (VEDF) ou eritropoietina (EPO)? Estas são questões a serem respondidas por pesquisas clínicas futuras.

Talvez o G-CSF possa ser um complemento ou possa ser associado ao implante de células mesenquimais. ${ }^{59} \mathrm{Num}$ trabalho recente, Baker, ${ }^{2}$ por exemplo, utilizou HSCs, inibidores da metaloproteinase e tratamento metabólico isoladamente ou em associação em animais. Num modelo de oclusão da ACM, vetores de liberação genéticos foram utilizados para potencializar a expressão de inibidores teciduais da matriz metaloproteinase 1 e 2 (TIMP1 e TIMP2) três dias antes da isquemia induzida. Após a oclusão, células HSCs foram injetadas por via intra-arterial isoladamente ou em combinação com agentes que melhoraram a biodisponibilidade de NO. A avaliação da extensão do infarto, a incorporação de BrdU e a recuperação motora demonstraram que os maiores benefícios foram obtidos nos ratos que receberam a terapia tripla combinada, que ultrapassaram os efeitos benéficos obtidos na monoterapia ou na dupla terapia.
Em outro estudo, Minger e colaboradores ${ }^{60}$ examina- $^{-}$ ram o cérebro de um paciente de 84 anos de idade que faleceu sete dias após um AVC utilizando-se anticorpos com marcadores específicos para células progenitoras neurais e as comparou com um cérebro na mesma idade e sexo. Os autores observaram a presença de células imunopositivas na região peri-infartada e também em áreas distantes. Todos os achados descritos em animais foram corroborados por este estudo anatomopatológico do cérebro humano.

No Brasil, apresentamos nossa experiência inicial com um paciente que talvez tenha sido o primeiro paciente brasileiro a fazer uso de G-CSF especificamente para uma patologia intracerebral, a qual se deu logo após a publicação de Shyu. ${ }^{38}$ Posteriormente a este caso, apresentamos um protocolo de pesquisa do G-CSF em pacientes com AVC na fase subaguda (15 a 90 dias), que está em análise pelo Conep.

\section{Conclusão}

A compreensão dos mecanismos envolvidos na plasticidade neural e na sua modulação, e a possibilidade de restauração de deficiências funcionais através da potencialização da neurogênese endógena ou da terapia celular abrem novos horizontes no tratamento do AVC. Estamos vivenciando uma nova era, e a próxima década possivelmente marcará o surgimento de novas tecnologias e abordagens para o tratamento do AVC e de outras doenças neurológicas.

\begin{abstract}
The granulocyte colony-stimulating-factor (G-CSF) is a glycoproteina which has been described for decades, and it is commonly utilized in the treatment of neutropenic states and bone marrow transplants. G-CSF stimulates hematopoietic stem-cels e crucially regulates the survival of mature neutrophils through a mechanism of apoptosis inhibition. Beyond its systemic effect, recently it has been shown its surprising activity in the central nervous system (CNS). G-CSF administration mobilizes bone marrow stem cells para systemic blood, and those cells cross the blood-brainbarrier e target brain's damaged area. G-CSF's activity in the CNS has been defined as multimodal, because additionally it has been demonstrated a direct neuroprotective action through different mechanisms such as antiapoptotic activity, angiogenesis, antiinflamatory effect, and stimulation of endogenous neurogenesis. This paper sumarizes G-CSF action in the CNS and approaches its potential para use in stroke. Rev. Bras. Hematol. Hemoter. 2009; 31(Supl. 1):104-111.
\end{abstract}

Key words: G-CSF; ischemia; brain ischemia; neuroprotection. 


\section{Referências Bibliográficas}

1. Araújo JD, Araújo Filho JD, Ciorlin E, Greco OT, Ardito RV, PironRuiz L, et al. Utilização de células-tronco de MO para tratamento de isquemia crítica de membro inferior. J Vasc Bras. 2006;5(3): 209-14.

2. Baker AH, Sica V, Work LM, Williams-Ignarro S, de Nigris F, Lerman $\mathrm{LO}$, et al. Brain protection using autologous bone marrow cell, metalloproteinase inhibitors, and metabolic treatment in cerebral ischemia.. Proc Natl Acad Sci USA. 2007;104(9):3597-602.

3. Bonn D. First cell transplant aimed to reverse stroke damage. Lancet. 1998;352(9122):119.

4. De Freitas GR, Mendonça MLF, Bezerra DC, Silva AS, Falcão CHE, Gonzales C, et al. Safety and feasibility of intra-arterial autologous bone marrow mononuclear cell transplantation in acute ischemic stroke. Stroke. 2006;37:624-5.

5. Greco OT, Ardito RV, Takeda RT, Lago MR, Poloni AFC, Jacob JLB, et al. Ressincronização cardíaca e terapia celular, alternativas para tratamento de pacientes com cardiomiopatia dilatada. Resultados preliminares. Reblampa. 2006;19(4):259-99.

6. Kondziolka D, Steinberg GK, Wechsler L, Meltzer CC, Elder E, Gebel J, et al. Neurotransplantation for patients with subcortical motor stroke: a phase 2 randomized trial. J Neurosurg. 2005;103 (1):38-45

7. Schneider A, Wysocki R, Pitzer C, Krüger C, Laage R, Schwab S, et al. An extended window of opportunity for G-CSF treatment in cerebral ischemia. BMC Biol. 2006;4:36.

8. Shyu WC, Lin SZ, Lee CC, Liu DD, Li H. Granulocyte colonystimulating factor for acute ischemic stroke: a randomized controlled trial. CMAJ. 2006;174(7):927-33.

9. Jin K, Minami M, Lan JQ, Mao XO, Batteur S, Simon RP, et al. Neurogenesis in dentate subgranular zone and rostral subventricular zone after focal cerebral ischemia in the rat. Proc Natl Acad Sci USA. 2001;98(8):4710-5.

10. Nakatomi H, Kuriu T, Okabe S, Yamamoto S, Hatano O, Kawahara $\mathrm{N}$, et al. Regeneration of hippocampal pyramidal neurons after ischemic brain injury by recruitment of endogenous neural progenitors. Cell. 2002;110(4):429-41.

11. Arvidsson A, Collin T, Kirik D, Kokaia Z, Lindvall O. Neuronal replacement from endogenous precursors in the adult brain after stroke. Nat Med. 2002;8(9):963-70.

12. Thored P, Arvidsson A, Cacci E, Ahlenius H, Kallur T, Darsalia $\mathrm{V}$, et al. Persistent production of neurons from adult brain stem cells during recovery after stroke. Stem Cells. 2006; 24(3):739-47.

13. Gibson CL, Bath PM, Murphy SP. G-CSF reduces infarct volume and improves functional outcome after transient focal cerebral ischemia in mice. J Cereb Blood Flow Metab. 2005;25 (4): 431-9.

14. Hirayama F, Yamaguchi M, Yano M, Yasui K, Horie Y, Matsumoto $\mathrm{K}$, et al. Spontaneous and rapid reexpression of functional CXCR4 by human steady-state peripheral blood CD34+ cells. Int J Hematol. 2003;78(1):48-55.

15. Hu B, Yasui K. Effects of colony-stimulating factors (CSFs) on neutrophil apoptosis: possible roles at inflammation site. Int J Hematol. 1997;66(2):179-88.

16. Jung KH, Chu K, Lee ST, Kang L, Kim SU, Kim M, et al. G-CSF protects human cerebral hybrid neurons against in vitro ischemia. Neurosci Lett. 2006;394(3):168-73.

17. Lee ST, Chu K, Jung KH, Ko SY, Kim EH, Sinn DI, et al. Granulocyte colony-stimulating factor enhances angiogenesis after focal cerebral ischemia. Brain Res. 2005;1058(1-2):120-8.

18. Lois C, García-Verdugo JM, Alvarez-Buylla A. Chain migration of neuronal precursors. Science. 1996;271(5251):978-81.
19. Lu CZ, Xiao BG. G-CSF and neuroprotection: a therapeutic perspective in cerebral ischaemia. Biochem Soc Trans. 2006;34(Pt 6):1327-33.

20. Schäbitz WR, Kollmar R, Schwaninger M, Juettler E, Bardutzky J, Schölzke MN, et al. Neuroprotective effect of granulocyte colonystimulating factor after focal cerebral ischemia. Stroke. 2003; 34(3):745-51.

21. Schneider A, Kuhn HG, Schäbitz WR. A role for G-CSF (granulocytecolony stimulating factor) in the central nervous system. Cell Cycle. 2005;4(12):1753-7.

22. Schneider A, Krüger C, Steigleder T, Weber D, Pitzer C, Laage R, et al. The hematopoietic factor G-CSF is a neuronal ligand that counteracts programmed cell death and drives neurogenesis. J Clin Invest. 2005;115(8):2083-98.

23. Begley CG, Lopez AF, Nicola NA, Warren DJ, Vadas MA, Sanderson $\mathrm{CJ}$, et al. Purified colony-stimulating factors enhance the survival of human neutrophils and eosinophils in vitro: a rapid and sensitive microassay for colony-stimulating factors. Blood. 1986; 68(1):162-6.

24. Burgess AW, Metcalf D. Characterization of a serum factor stimulating the differentiation of myelomonocytic leukemic cells. Int J Cancer. 1980;26(5):647-54.

25. Morstyn G, Campbell L, Lieschke G, Layton JE, Maher D, O'Connor $\mathrm{M}$, et al. Treatment of chemotherapy-induced neutropenia by subcutaneously administered granulocyte colony-stimulating factor with optimization of dose and duration of therapy. J Clin Oncol. 1989;7(10):1554-62.

26. Nicola NA, Begley CG, Metcalf D. Identification of the human analogue of a regulator that induces differentiation in murine leukaemic cells. Nature. 1985;314(6012):625-8.

27. Fukunaga R, Seto Y, Mizushima S, Nagata S. Three different mRNAs encoding human granulocyte colony-stimulating factor receptor. Proc Natl Acad Sci USA. 1990;87(22):8702-6.

28. Layton JE, Hall NE, Connell F, Venhorst J, Treutlein HR. Identification of ligand-binding site III on the immunoglobulinlike domain of the granulocyte colony-stimulating factor receptor. J Biol Chem. 2001;276(39):36779-87.

29. Welte K, Platzer E, Lu L, Gabrilove JL, Levi E, Mertelsmann R, et al. Purification and biochemical characterization of human pluripotent hematopoietic colony-stimulating factor. Proc Natl Acad Sci USA. 1985;82(5):1526-30.

30. Nagata S, Tsuchiya M, Asano S, Kaziro Y, Yamazaki T, Yamamoto $\mathrm{O}$, et al. Molecular cloning and expression of cDNA for human granulocyte colony-stimulating factor. Nature. 1986;319(6052): 415-8.

31. Neidhart J, Mangalik A, Kohler W, Stidley C, Saiki J, Duncan P, et al. Granulocyte colony-stimulating factor stimulates recovery of granulocytes in patients receiving dose-intensive chemotherapy without bone marrow transplantation. J Clin Oncol. 1989;7(11): 1685-92.

32. Sheridan WP, Morstyn G, Wolf M, Dodds A, Lusk J, Maher D, et al. Granulocyte colony-stimulating factor and neutrophil recovery after high-dose chemotherapy and autologous bone marrow transplantation. Lancet. 1989;2(8668):891-5.

33. Vallières L, Campbell IL, Gage FH, Sawchenko PE. Reduced hippocampal neurogenesis in adult transgenic mice with chronic astrocytic production of interleukin-6. J Neurosci. 2002;22(2): 486-92.

34. Gibson CL, Jones NC, Prior MJ, Bath PM, Murphy SP. G-CSF suppresses edema formation and reduces interleukin-1beta expression after cerebral ischemia in mice. J Neuropathol Exp Neurol. 2005;64(9):763-9.

35. Kawada H, Takizawa S, Takanashi T, Morita Y, Fujita J, Fukuda K, et al. Administration of hematopoietic cytokines in the subacute 
phase after cerebral infarction is effective for functional recovery facilitating proliferation of intrinsic neural stem/progenitor cells and transition of bone marrow-derived neuronal cells. Circulation. 2006;113(5):701-10.

36. Komine-Kobayashi M, Zhang N, Liu M, Tanaka R, Hara H, Osaka A, et al. Neuroprotective effect of recombinant human granulocyte colony-stimulating factor in transient focal ischemia of mice. $\mathrm{J}$ Cereb Blood Flow Metab. 2006;26(3):402-13.

37. Shyu WC, Lin SZ, Yang HI, Tzeng YS, Pang CY, Yen PS, et al. Functional recovery of stroke rats induced by granulocyte colonystimulating factor-stimulated stem cells. Circulation. 2004;110 (13): 1847-54.

38. Six I, Gasan G, Mura E, Bordet R. Beneficial effect of pharmacological mobilization of bone marrow in experimental cerebral ischemia. Eur J Pharmacol. 2003;458(3):327-8.

39. Hartung T. Anti-inflammatory effects of granulocyte colonystimulating factor. Curr Opin Hematol. 1998;5(3):221-5.

40. Konishi Y, Chui DH, Hirose H, Kunishita T, Tabira T. Trophic effect of erythropoietin and other hematopoietic factors on central cholinergic neurons in vitro and in vivo. Brain Res. 1993;609(12):29-35.

41. Yanqing Z, Yu-Min L, Jian Q, Bao-Guo X, Chuan-Zhen L. Fibronectin and neuroprotective effect of granulocyte colonystimulating factor in focal cerebral ischemia. Brain Res. 2006; 1098(1):161-9.

42. Harada M, Qin Y, Takano H, Minamino T, Zou Y, Toko H, et al. G-CSF prevents cardiac remodeling after myocardial infarction by activating the Jak-Stat pathway in cardiomyocytes. Nat Med. 2005;11(3):305-11.

43. Sakai T, Johnson KJ, Murozono M, Sakai K, Magnuson MA, Wieloch $\mathrm{T}$, et al. Plasma fibronectin supports neuronal survival and reduces brain injury following transient focal cerebral ischemia but is not essential for skin-wound healing and hemostasis. Nat Med. 2001;7(3):324-30.

44. Ferrer I, Planas AM. Signaling of cell death and cell survival following focal cerebral ischemia: life and death struggle in the penumbra. J Neuropathol Exp Neurol. 2003;62(4):329-39.

45. Ohki Y, Heissig B, Sato Y, Akiyama H, Zhu Z, Hicklin DJ, et al. Granulocyte colony-stimulating factor promotes neovascularization by releasing vascular endothelial growth factor from neutrophils. FASEB J. 2005;19(14):2005-7.

46. Takagi Y, Omura T, Yoshiyama M, Matsumoto R, Enomoto S, Kusuyama $\mathrm{T}$, et al. Granulocyte-colony stimulating factor augments neovascularization induced by bone marrow transplantation in rat hindlimb ischemia. J Pharmacol Sci. 2005;99(1):45-51.

47. Minamino K, Adachi $\mathrm{Y}$, Okigaki M, Ito $\mathrm{H}$, Togawa $\mathrm{Y}$, Fujita $\mathrm{K}$, et al. Macrophage colony-stimulating factor (M-CSF), as well as granulocyte colony-stimulating factor (G-CSF), accelerates neovascularization. Stem Cells. 2005;23(3):347-54

48. Görgen I, Hartung T, Leist M, Niehörster M, Tiegs G, Uhlig S, et al. Granulocyte colony-stimulating factor treatment protects rodents against lipopolysaccharide-induced toxicity via suppression of systemic tumor necrosis factor-alpha. J Immunol. 1992;149(3): 918-24.

49. Squadrito F, Altavilla D, Squadrito G, Campo GM, Ioculano M, Ammedolia L, et al. The effects of recombinant human granulocyte-colony stimulating factor on vascular dysfunction and splanchnic ischaemia-reperfusion injury. Br J Pharmacol. 1997;120(2):333-9.

50. Heard SO, Fink MP. Counterregulatory control of the acute inflammatory response: granulocyte colony-stimulating factor has anti-inflammatory properties. Crit Care Med. 1999;27(5): 1019-21.
51. Ekdahl CT, Claasen JH, Bonde S, Kokaia Z, Lindvall O. Inflammation is detrimental for neurogenesis in adult brain. Proc Natl Acad Sci USA. 2003;100(23):13632-7.

52. Gebicke-Haerter PJ. Microglia in neurodegeneration: molecular aspects. Microsc Res Tech. 2001;54(1):47-58.

53. Hanisch UK. Microglia as a source and target of cytokines. Glia. 2002;40(2):140-55

54. Pocock JM, Liddle AC. Microglial signalling cascades in neurodegenerative disease. Prog Brain Res. 2001;132:555-65.

55. Monje ML, Toda H, Palmer TD. Inflammatory blockade restores adult hippocampal neurogenesis. Science. 2003; 302 (5651): 1760-5.

56. Kondziolka D, Wechsler L, Goldstein S, Meltzer C, Thulborn KR, Gebel J, et al. Transplantation of cultured human neuronal cells for patients with stroke. Neurology. 2000;55(4):565-9.

57. Ickenstein G, Haas S, Sauerbruch S, et al. Regeneration in acute ischemic stroke (RAIS) with hematopoietic stem cell mobilization (abstract) In 56th Annual Meeting of the American Academy of Neurology; 2004, Program No S39.001.

58. Sprigg N, Bath PM, Zhao L, Willmot MR, Gray LJ, Walker MF, et al. Granulocyte-colony-stimulating factor mobilizes bone marrow stem cells in patients with subacute ischemic stroke. Stroke. 2006;37(12):2979-83.

59. Mendez-Otero R, de Freitas GR, André C, de Mendonça ML, Friedrich M, Oliveira-Filho J. Potential roles of bone marrow stem cells in stroke therapy. Regen Med. 2007;2(4):417-23.

60. Minger SL, Ekonomou A, Carta EM, Chinoy A, Perry RH, et al. Endogenous neurogenesis in the human brain following cerebral infarction. Regen Med. 2007;2(1):69-74.

Avaliação: O tema apresentado consta da pauta elaborada pelo editor, Professor Milton Artur Ruiz e coeditores deste suplemento, Professores Sergio Paulo Bydlowski e Adriana Seber.

Conflito de interesse: não declarado

Recebido: 30/06/2008

Aceito: $03 / 11 / 2008$ 